\title{
ESTIMATION METHOD OF LEFT TURN LANE CAPACITY UNDER THE INFLUENCE OF PEDESTRIANS AND CROSSWALK LAYOUT AT SIGNALIZED CROSSWALKS
}

\author{
Yonas M. Emagnua ${ }^{\star}$, Xin Zhang ${ }^{\mathrm{a}}$, Miho Iryo-Asano ${ }^{\mathrm{a}}$ and Hideki Nakamura ${ }^{\mathrm{a}}$
}

\begin{abstract}
The left turn lane capacity is highly influenced by the presence of pedestrians under shared signal phasing. Pedestrian crossing time can be highly influenced by pedestrian platoon maneuver under the influence of traffic signal settings, crosswalk length and bidirectional interactions of pedestrian, due to that left turning vehicles movement may be significantly affected. However, existing capacity estimation methods of left turn lanes did not consider those factors. In addition, the influence of crosswalk layout did not properly consider in the existing methods and researches. This study aims to propose a method to estimate the left turn lane capacity, considering the characteristics of crossing pedestrians and crosswalk layout variations. Waiting pedestrian presence-time is estimated by applying pedestrian presence probability model. In addition, the left turning vehicles discharge flow rate under different crosswalk layout and pedestrian demand condition are investigated and modeled. The left turn lane capacity estimation equation is proposed by characterizing the observed discharge flow rate of left turning vehicles under the influence of crossing pedestrians. It was found that the proposed capacity estimation method can represent the realistic influence of pedestrians and crosswalk layout on the left turn lane capacity at signalized crosswalks.
\end{abstract}

Keywords: Signalized crosswalk, capacity, pedestrian presence, crosswalk layout, discharge flow rate.

\section{INTRODUCTION}

The two main users at signalized intersections are vehicles and pedestrians. Based on the assigned signal timing design they may share space and time in their movement around the signalized crosswalks. Most of the time, traffic engineers are assigning concurrent signal timing design for crossing pedestrians and turning vehicles and facilitate the smooth traffic movement at signalized crosswalks by minimizing delay. Therefore, understanding vehiclepedestrian interaction is significant in concurrent signal timings. One example for pedestrian-vehicle interaction is when left turning vehicles (left-hand traffic system) and crossing pedestrians have shared signal timing interval.

Due to the interaction situation the operational performance of left turning vehicles movement is highly influenced by crossing pedestrians. The longest interaction time interval between crossing pedestrians and left turning vehicles is happening when the platoon of waiting pedestrians crosses the crosswalk, at this time interval left turning vehicles are blocked by crossing pedestrians significantly. Note that Japan has a left-hand traffic system. All of the following analysis and discussions are for the left-hand traffic.

Capacity is one of the methods applied to measure the performance of signalized intersection. If the capacity estimation is not realistic then it will be difficult to have a proper signal timing design, consequently a problem of delay and congestion may happen at the signalized intersections. Accordingly, to estimate the left turn lane capacity at signalized intersections the proper consideration of crossing pedestrians' influence on left turning vehicles movement is necessary. In addition to crossing pedestrians' characteristics the geometric layout of the signalized crosswalk may influence the left turn lane capacity estimation.

Therefore, the objective of this paper is to propose a

a Graduate School of Environmental Studies, Nagoya University, Furocho, Chikusa-ku, Nagoya 464-8603, Japan.

*Corresponding author email address: yoethio2003@gmail.com method to estimate the left turn lane capacity at signalized crosswalks by considering the influence of pedestrian characteristics and geometric layout of the crosswalk.

\section{LITERATURE REVIEW}

\section{A. INFLUENCE OF PEDESTRIANS ON LEFT TURN LANE CAPACITY}

The existing guidelines or researches do not fully consider different influencing factors on pedestrian and turning vehicles flows for estimating turning vehicle capacity. For instance, Highway Capacity Manual [1] and a Planning and Design of at-grade Intersections - Basic Edition -; Guide for Planning, Design and Traffic Signal Control of Japan (JSTE manual, here after) [2] considered the influence of pedestrians for estimating capacity of turning lanes. The basic idea to consider the pedestrian impact is based on the occupancy of crossing pedestrians within green time. Their estimation of the pedestrian influence adjustment factor is mostly related with pedestrian volume. Lu et al [3] investigated the influence of pedestrians on the right-turn lane capacity (right-hand traffic system) by using the parking line method, acceptable gap theory, and video survey. Their results showed that the traffic volume of pedestrian has a significant impact on the traffic capacity of right turning motor vehicles. Zhang et al [4] quantitatively analyzed pedestrian impact on the capacity of right-turn vehicles (right-hand traffic system) at signalized intersections.

They applied pedestrian-grouping model based on gap acceptance theory to estimate potential capacity. In addition, based on their statistical analysis, they concluded that turning vehicles saturation flow rate is mainly affected by the position of pedestrian in the crosswalk (inside or outside of left-turn vehicle's trajectory), and the distance between the vehicle and pedestrians. However, in the manuals and also other studies use the number of pedestrians as a factor to investigate the influence of pedestrians on left turning vehicles without considering the bi-directional pedestrian 
flow interaction, pedestrian platoon effect and change in geometric layout of the crosswalk.

\section{B. IMPACT OF PEDESTRIAN ON SATURATION FLOW RATE}

Chen et al [5] studied impact of pedestrian traffic on saturation flow of left-turn at urban intersections; they found that $93 \%$ of left-turn vehicles slowed down due to the presence of pedestrians. Roshani and Bargegol [6] investigated the effect of pedestrians on the saturation flow rate of right turn (right-hand traffic system) movements at signalized intersection in Iran. They did an empirical observation and model the relation between pedestrian flow and turning vehicles flow. Their results indicated that there is a primarily linear relationship between pedestrian volume and right turning vehicles flow. Nassereddine et.al [7] investigated the pedestrianvehicle interaction and tried to establish a simple nonprobabilistic regression model that explains the attitude of right-turning drivers (right-hand traffic system) at a specific intersection towards the presence of conflicting pedestrians. Their result confirmed that, when drivers perceive the possibility of pedestrian reaching a critical conflict point at the same time as them, they will modify their behavior even if not coming to a stop. Therefore, they argued that these interaction modeling can be used to evaluate the effectiveness of traffic signal control.

In addition, the crosswalk layout may influence the behavior of crossing pedestrians and left turning vehicles saturation flow rate at the signalized crosswalk. Iasmin et al [8] studied the yielding behavior of left turning vehicles for pedestrians under different intersection angle conditions. Their analysis showed that turning vehicles behavior at obtuse angle intersections is more nonyielding than other intersections. Consequently, there will be variation in saturation flow rate of the turning lane.

\section{CROSSING PEDESTRIAN'S BEHAVIOR}

In the study of left turn lane capacity estimation under concurrent signal timing of crossing pedestrians with left turning vehicles, proper understanding of crossing pedestrians' behavior is necessary. The influence of pedestrians on left turning vehicles movement is highly related with the crossing behavior of pedestrians. A research by Akash et al [9] on the analysis of pedestrian crossing behavior by considering pedestrian characteristics like age, gender and that of carrying baggage influence on crossing speed and waiting time showed that there is a significant variation in waiting time and crossing speed due to the difference in the composition of crossing pedestrians. Crossing speed is other important influencing parameter for crossing pedestrian behavior. Muley et al [10] analyzed three types of pedestrian speeds (entry speed, crossing speed, and exit speed) at signalized crosswalks and their results showed that the pedestrian entry speeds were significantly affected by the pedestrian signal indications. In addition, the crossing speeds were positively correlated with crosswalk length for pedestrians crossing on green and red indications while pedestrian exit speeds were independent of crosswalk length but significantly affected by crossing direction. Park et al [11] investigated pedestrian crossing speed at signalized intersections with heavy pedestrian volumes. They attempted to model the relationships between pedestrian speeds and densities, and between speeds and directional proportions of pedestrian flows based on signalized intersections with heavy pedestrian volumes. Their findings showed that pedestrian crossing speeds within high density crosswalks are significantly affected by opposing pedestrian flows.

\section{PEDESTRIAN PRESENCE-TIME}

At the onset of green, pedestrians waiting at sidewalks simultaneously starts to cross from both side of the crosswalks. Generally speaking, these pedestrians forming platoons at the onset of green significantly contribute to block turning vehicle flows. Consequently, in order to propose a method for estimating expected blockage time of left turning vehicles due to pedestrians at signalized crosswalks, the pedestrian presence probability model proposed by Zhang and Nakamura [12] is adjusted by the authors of this paper by considering the interaction of subject and opposing pedestrian flow [13].The result showed that for the coefficients $\alpha$ and $\beta$ of the Weibull distribution pedestrian presence probability model, elapsed time has a positive impact to both $\alpha$ and $\beta$, which shows pedestrian platoon dispersed in the direction of flow when elapsed time increases. Crosswalk length has a negative impact to $\alpha$, suggesting that there are the wider distributions along the longer crosswalk. On the longer crosswalks, pedestrian platoon tends to disperse. In addition, the re-estimated model revealed that under bidirectional pedestrian flow the high demand of opposing pedestrians will impede the pedestrian platoon to disperse. The re-estimated model showed a good fitting for high pedestrian demand crosswalks compared with Zhang's model. With the assumption of waiting pedestrians influence left turning vehicles significantly, the reestimated model is used to estimate the expected waiting pedestrian presence-time.

Therefore, for estimation of left turn lane capacity at signalized crosswalks a proper methodology which consider pedestrian presence at the crosswalk at higher and lower pedestrian demand condition and easy to use in macroscopic level under different geometric layout of the crosswalk is necessary.

\section{METHODOLOGY}

\section{A. DEFINITIONS OF TERMINOLOGIES}

Figure 1 shows the layout of a signalized crosswalk. In this paper, a coordinate transformation of pedestrian position is conducted referring to the edge of crosswalk. The horizontal axis $\mathrm{x}$ is defined parallel to the edge of cyclist pathway (which is located at the right side of the crosswalk from the near-side direction) and the y-axis position of pedestrians is projected along the horizontal axis. Since the moving distance on the horizontal axis is more important for understanding the pedestrian influence on left turning vehicles, only the pedestrian movement along the horizontal axis is considered. In this study the discharge flow of left turning vehicles is measured at the edge of the crosswalk located opposite to the $\mathrm{x}$-axis as shown in Figure 1.The travel time of left turning vehicles 
from the stop line up to the discharge flow measurement section is affected by stop-line and crosswalk setback distances. As our target is to understand the influence of crossing pedestrians on the left turning vehicles movement, the observation must be after the vehicles pass the shared space.

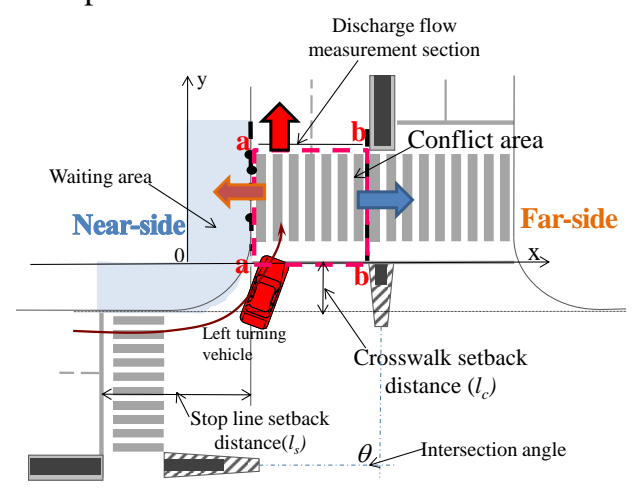

Figure 1 Layout of subject crosswalk and definitions of basic terms

- Crossing pedestrians at signalized intersection are classified by arrival timing in two groups:

a. Waiting pedestrians: are pedestrians who arrived at the crosswalk before the onset of pedestrian green time.

b. Arriving pedestrians: are pedestrians who arrive at the crosswalk after the onset of pedestrian green time.

- Conflict-area: is a shared space by crossing pedestrians and turning vehicles on the crosswalk as shown in Figure1.

- Near-side pedestrians: are coming from the nearest side of the crosswalk to left turning vehicles.

- Far-side pedestrian: are coming from the opposite side from the near-side pedestrian flow and this is the farthest side of crosswalk for left turning vehicles.

- Expected waiting pedestrian presence-time (EPT): is the expected time interval when waiting pedestrians platoon occupied the conflict-area.

- Last left turning vehicle: is the last vehicle passing the measurement cross-section in the group of left turning vehicles under continues flow condition. th is the measurement cross-section passing time of the last vehicle in the group of continues left turning vehicles that started from the onset of green.

- Pedestrian green time (PG): is the time interval assigned for crossing pedestrians from both sides of the crosswalk.

- Pedestrian flash green time (PFG): is the time interval arriving pedestrian are not allowed to enter into the crosswalk, however pedestrians who are still on the crosswalk have to finish crossing within this time interval.

- Green time $(\mathrm{G})$ : is assigned green time for left turning vehicles.

As it is shown in Figure 2 within the assigned concurrent signal timing we may have two portions (EPT and left turning vehicles discharge time) of time intervals. The time intervals are proposed based on the crossing pedestrians and left turning vehicles movement characteristics. At the onset of PG, a group of waiting pedestrians start to pass the conflict-area and when the last pedestrians from near-side/far-side clear from the conflict-area the first time interval portion will finish.

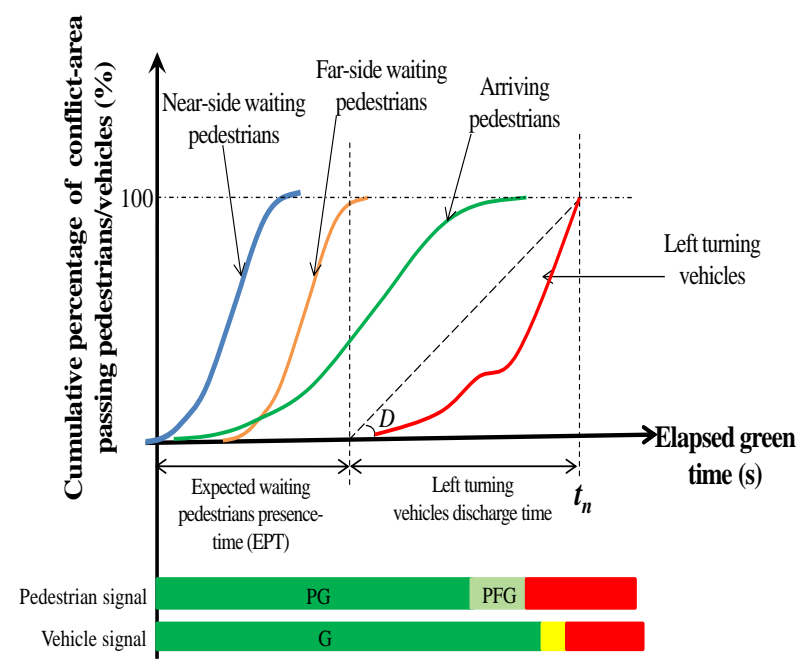

Figure 2 Pedestrian and left turning vehicle conflict-area passing distribution

Arriving pedestrians can enter the crosswalk at any time after the onset of green until they clear in the PFG time; meanwhile left turning vehicles may pass the crosswalk by searching acceptable gap between arriving pedestrians. Therefore, the second time interval portion is the left turning vehicles discharge flow time by interacting with arriving pedestrians.

\section{B. EXPECTED WAITING PEDESTRIAN PRESENCE -TIME (EPT)}

The first time interval portion is from the onset of green until the last near-side/far-side waiting pedestrian exit from the conflict-area. This is the time interval that waiting pedestrians platoon highly influence the left turning vehicles movement. As it is defined in a study by Emagnu et al. [13] to estimate the expected waiting pedestrian presence-time at the conflict-area of signalized crosswalks, it is necessary to consider the clearance time of pedestrian from both sides of the crosswalk at the edges of the conflict-area as shown in Figure 1.

Figure 3 depicts the concept of how to calculate expected durations that waiting pedestrian platoon blocks the conflict area using the pedestrian probability distribution. The upper part of figure in Figure 3 shows the time dependent trend of cumulative pedestrian presence probability distribution of near-side pedestrians. Let us define $q_{n}$ as the number of near-side waiting pedestrians per cycle. As the time goes, the distribution moves toward right-hand side. In this figure, at time $t=$ $t_{n \_e n}$, the cumulative probability at cross section $a\left(x=x_{a}\right)$ will be $\theta_{n \_e n}$. This means that the expected number of (1$\theta_{n \_ \text {en }} q_{n}$ has been already passed the cross section $a$ by that time. Suppose this expected number is equal to one as in Eqn. (1), by assuming the entry of the first waiting pedestrian. Then, the time $t_{n \_ \text {en }}$ will be considered as the expected time that first pedestrian in the platoon arrives at the cross-section $a$. 


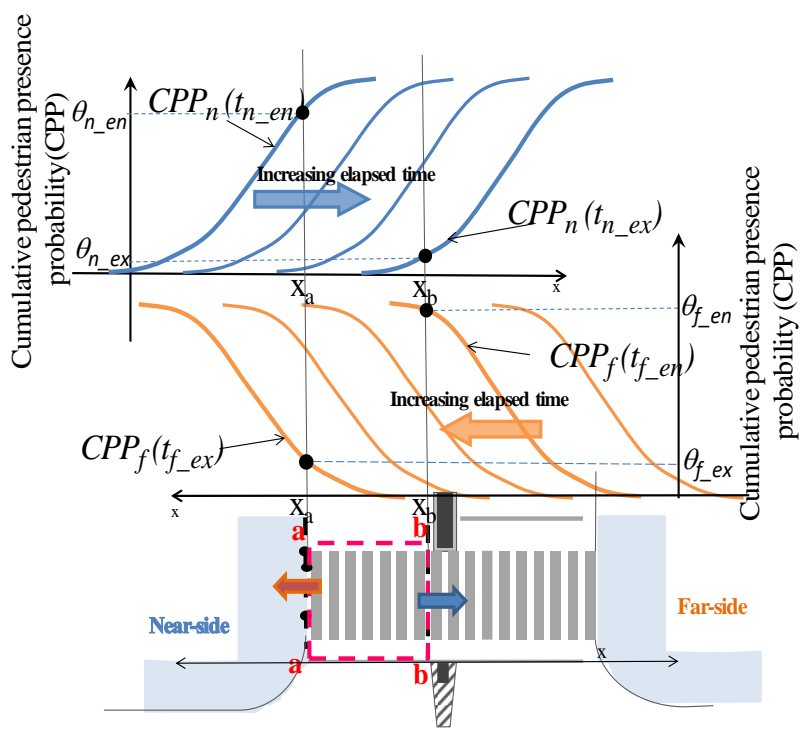

Figure 3 Pedestrian crossing probability of conflict-area

$$
\left(1-\theta_{n \_ \text {en }}\right) q_{n}=1
$$

Actually, $\theta_{n \_e n}, x_{a}$ are given from the definition but $t_{n \_ \text {en }}$ is the unknown variable. Therefore, the problem to be solved is to find out $t_{n_{-} e n}$ so that Equation (1) and (2) are satisfied.

$$
\theta_{n \_ \text {en }}=\int_{0}^{t_{n} \text { en }} P P P_{n}\left(x_{a}, t\right) d t
$$

Pedestrian presence probability (PPP) is modeled by (Emagnu et.al. 2020) as a function of elapsed time as explained in the previous section. However, it is difficult to analytically solve the right-hand side of Eqn. (2). Therefore, numerical calculation is applied to obtain $t_{n \_} e n$.

Similarly, time $t_{n \_e x}$, i.e. the expected exit time of the last near-side pedestrian from conflict area, is given by satisfying Equation (4) and (5).

$$
\begin{gathered}
\theta_{n \_ \text {ex }} \times q_{n}=1 \\
\theta_{n \_ \text {ex }}=\int_{0}^{t_{n} e x} P P_{n}\left(x_{b}, t\right) d t
\end{gathered}
$$

Where, $\theta_{n \_ \text {ex }}$ is cumulative probability at cross section $b(x$ $\left.=x_{b}\right)$.

Entering $\left(t_{f_{-} e n}\right)$ and exit time $\left(t_{f_{-} e x}\right)$ of far-side pedestrian platoons are also derived by using Figure 3 following the same approach. Thus, after the expected entry/exit times of both near-side and far-side platoons are obtained, total estimated expected pedestrian presencetime at the conflict area of signalized crosswalk, (Hereafter, expected waiting pedestrian presence-time (EPT), will be calculated by Equation (5).

$$
E P T=\min \left(\begin{array}{c}
\left(t_{f_{-} e x}-t_{n_{-} e n}\right), \\
\left(t_{f_{-} e x}-t_{n_{-} e n}\right)+\left(t_{f_{-} e x}-t_{n_{-} e n}\right)
\end{array}\right)
$$

Pedestrian presence-time may have two values as shown in Equation (5) and the selection of the minimum value depends on crosswalk length, pedestrian demand and assigned signal timing. These factors influence the overlap time of the last pedestrian from near-side direction flow and first pedestrian of far-side direction flow.

\section{LEFT TURNING VEHICLES DISCHARGE TIME}

Left tuning vehicles discharge time is defined as the expected time after the waiting pedestrian platoon clear from the conflict-area until the last queued left turning vehicle pass the conflict area (the observation section is the edge of the conflict-area along the left turning vehicles movement direction as shown in Figure 1). In the cumulative distribution of number of left turning vehicles passing the conflict-area as shown in Figure 2, the slope of the line which connects the end of EPT and $t_{n}$ is the discharge flow rate $(D)$ of the left turning vehicles movement within available discharge time under the influence of arriving pedestrians. Left turning vehicle discharge flow rate can be influenced by the pedestrian arrival rate and the geometric layout of the crosswalk and intersection, like crosswalk setback distance, intersection angle, stop-line setback distance and crosswalk length. To estimate the discharge flow rate of left turning vehicles, observation of the conflict-area passing time of the last queued vehicle is necessary. Therefore, discharge flow rate for each observed cycle can be estimated using Equation (6).

$$
D=3600 *\left(\frac{n}{t_{n}-E P T}\right)
$$

Where, $D$ is the discharge flow rate (veh/hr), $n$ is the number of discharged left turning vehicles, $t_{n}$ is crosswalk exit time of the last queued left turning vehicle and EPT is the observed waiting pedestrian presence-time (s).

\section{LEFT TURN LANE CAPACITY ESTIMATION}

Left turning vehicles pass the conflict-area based on the condition of crossing pedestrians on the crosswalk. At the onset of green the presence probability of near-side waiting pedestrians on the conflict-area is high, since near-side waiting pedestrians' waiting area is near to the conflict-area compared with left turning vehicles and farside waiting pedestrians. After that, far-side waiting pedestrians join the near-side pedestrians or arrive to the conflict area after near-side waiting pedestrians clear from the conflict-area.

Meanwhile, arriving pedestrians may presence on the conflict area any time after the onset of green. Therefore, the maximum number of left turning vehicles that can pass the conflict-area within the assigned green time is highly influenced by the interaction with crossing pedestrians. As it is discussed in the above sections, the characteristics of waiting pedestrian flow and the interaction of arriving pedestrians on left turning vehicles can be empirically observed and modeled to capture the influence of crossing pedestrians on left turning vehicle movements. After we understand the crossing pedestrian's characteristics and the discharge flow rate, by considering the pedestrians behavior and geometric layout of the crosswalk, the left turn lane capacity can be estimated using Equation (7). 
Table 1 Conditions of observed sites

\begin{tabular}{|c|c|c|c|c|c|c|c|c|}
\hline \multicolumn{3}{|c|}{ Intersection name } & Kanayama & Ueda & Nishiosu & Otsu-dori & $\begin{array}{c}\text { Mizuho- } \\
\text { kuyakushyo* }\end{array}$ & Imaike \\
\hline \multicolumn{3}{|c|}{ Subject crosswalk } & East & South & North & West & East & East \\
\hline \multicolumn{3}{|c|}{ PG (s) } & $48-61$ & $47-62$ & 38 & 37 & 58 & 45 \\
\hline \multirow{3}{*}{ Signal phase } & \multicolumn{2}{|c|}{$\mathrm{G}(\mathrm{s})$} & $57-71$ & $61-67$ & 52 & 54 & 70 & 60 \\
\hline & \multicolumn{2}{|c|}{ PFG (s) } & 6 & 8 & 8 & 9 & 4 & 8 \\
\hline & \multicolumn{2}{|c|}{ Cycle length (s) } & $148-174$ & $144-176$ & 160 & 160 & 140 & 160 \\
\hline \multirow{4}{*}{$\begin{array}{c}\text { Waiting } \\
\text { pedestrians } \\
\text { (ped/cycle) }\end{array}$} & \multirow{2}{*}{ Near-side } & Range & $0-13$ & $0-3$ & $0-4$ & $1-13$ & $3-25$ & $1-6$ \\
\hline & & Average & 5 & 2 & 3 & 6 & 9 & 4 \\
\hline & \multirow{2}{*}{ Far-side } & Range & $0-10$ & $0-4$ & $0-3$ & $2-12$ & $1-5$ & $2-9$ \\
\hline & & Average & 4 & 2 & 2 & 5 & 3 & 5 \\
\hline \multirow{4}{*}{$\begin{array}{c}\text { Arriving } \\
\text { pedestrians } \\
\text { (ped/cycle) }\end{array}$} & \multirow{2}{*}{ Near-side } & Range & $0-6$ & $0-2$ & $0-3$ & $0-3$ & $4-9$ & $1-4$ \\
\hline & & Average & 3 & 1 & 2 & 2 & 7 & 3 \\
\hline & \multirow{2}{*}{ Far-side } & Range & $0-3$ & $0-5$ & $0-2$ & $1-6$ & $0-1$ & $1-3$ \\
\hline & & Average & 2 & 2 & 1 & 3 & 1 & 2 \\
\hline \multirow{2}{*}{$\begin{array}{l}\text { Crosswalk } \\
\text { geometry }\end{array}$} & \multirow{2}{*}{\multicolumn{2}{|c|}{$\begin{array}{l}\text { Length }(\mathrm{m}) \\
\text { Width }(\mathrm{m})\end{array}$}} & 16 & 21 & 32 & 34 & 10 & 21 \\
\hline & & & 6 & 5 & 6 & 6 & 6 & 7 \\
\hline \multicolumn{3}{|c|}{ Intersection angle $\theta$ (degree) } & 93 & 124 & 78 & 91 & 93 & 100 \\
\hline \multicolumn{3}{|c|}{ Setback distance of LTV stop line $l s(\mathrm{~m})$} & 21.4 & 16.5 & 17.5 & 21.5 & 13.5 & 28 \\
\hline \multicolumn{3}{|c|}{$\begin{array}{l}\text { Setback distance of subject crosswalk } l c \\
(\mathrm{~m})\end{array}$} & 4 & 20.1 & 13.1 & 12.5 & 1.5 & 14.5 \\
\hline \multicolumn{3}{|c|}{ Number of sample cycles for LTV analysis } & 14 & 24 & 27 & 19 & 4 & 9 \\
\hline
\end{tabular}

*Mizuho-kuyaushyo here in after named as Mizuho.

$$
c=\frac{(G-E P T)^{*} D\left(X_{p}, X_{c}\right)}{C}
$$

Where, $c$ is capacity of left turn lane (veh/hr), $G$ is green time (s), EPT is expected waiting pedestrian-presence time (s), $D$ is discharge flow rate (veh/hr), $X p$ is the influence of pedestrians, $X c$ is the influence of crosswalk layout and $C$ is cycle length (s).

\section{DATA COLLECTION}

\section{A. STUDY SITES AND TRAFFIC CONDITIONS}

For the discharge flow rate analysis and the left turn lane capacity estimation, six signalized intersections located in Nagoya, Japan are selected and the movement of pedestrians and vehicles are video recorded in the weekdays, considering the peak hour and under sunny weather condition. The intersections have different pedestrian demand situations and crosswalk geometric characteristics. The geometric condition, traffic condition and signal settings of the selected signalized crosswalks are summarized in Table 1.

The positions of pedestrians when they cross along the crosswalk and when the rear wheel of the left turning vehicles start from the stop line until it passes the conflictarea at every $0.1 \mathrm{~s}$ are manually extracted from observation videos by using the image processing system Traffic Analyzer [14] then the coordinates in these images are converted to global coordinate through the projective transformation. Kalman smoothing method is applied to estimate trajectories of left turning vehicles at each time interval. The observed cycles are selected by considering certain constraints that may affect the discharge flow rate i.e. only passenger cars, no impact of bicycles and no impact of heavy vehicles. All observed cycles had continues left turning vehicles flow at the onset of green, with a minimum five number of lined up left turning vehicles.

\section{ANALYSIS AND DISCUSSION}

\section{A. OBSERVED LEFT TURNING VEHICLES DISCHARGE FLOW}

Figure 4 show example cycles of observed left turning vehicles passing time distribution within available green time for Kanayama crosswalk. The distribution curves located in different horizontal position and also they have different slope $(D)$, which implies that for the observed cycles the influence of pedestrians and the response of left turning vehicles may significantly fluctuate when we compare observed cycles. As shown in Figure 4, from distribution curves we can notice that the first left turning vehicle passing time is shifted at least $15 \mathrm{sec}$ after the onset of green, two factors may contribute for this; the first one is the influence of waiting pedestrians i.e. waiting pedestrians may block the movement of left turning vehicles and the second one is related with geometric layout of the crosswalk i.e. if the position of stop line for the left turning vehicles is far from the crosswalk it takes time to arrive at the subject crosswalk before they pass the conflict-area.

In Figure 4 the red color distribution curve represents an observed cycle without arriving pedestrian presence. As it is shown in the figure, the position of the red curve is in the front position and the curve slope is relatively steep compared with the others. This indicate that, if there are no arriving pedestrians left turning vehicles start to discharge in short time after the waiting pedestrians clear from the conflict-area. The green color distribution curve represents an observed cycle in which arriving pedestrians enter only from near-side direction. Therefore, the position of the curve is shifted to the right from the red curve and the slope is similar with the red curve, because 
only near-side arriving pedestrians influence the left turning vehicles. The other curves (black and blue) characterize an observed cycle with both directional arriving pedestrians. Their position and slope are different because of the variance in the number of arriving pedestrians per cycle and directional composition of arriving pedestrians.

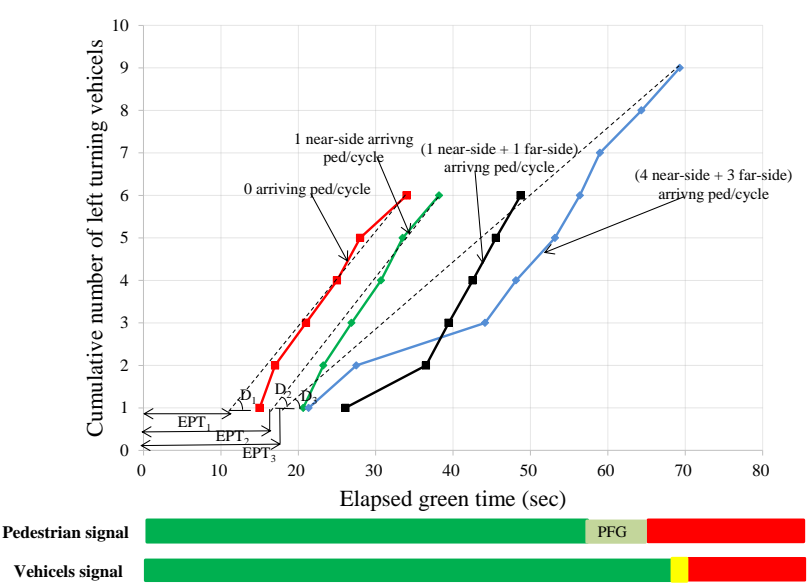

Figure 4 Cumulative numbers of left turning vehicles for Kanayama crosswalk

\section{B. MODELING LEFT TURNING VEHICLES DISCHARGE FLOW RATE}

After the observation of left turning vehicles passing time distribution the discharge flow rate is computed using Equation (6) for all observed cycles. Figure 5 and Figure 6 shows examples of the discharge flow rate related with pedestrian arrival rate of Kanayama and Otsu-dori crosswalks. For Kanayama crosswalk 16 cycles $(n=16)$ are observed, while for Otsu-dori crosswalk 19 cycles $(\mathrm{n}=19)$ are observed. In Figure 5 and Figure $6 r$ represent the correlation coefficient between discharge flow rate and pedestrian arrival rate. For both crosswalks, the discharge flow rate has a decreasing trend with increase in near-side and far-side pedestrian arrival rates, as the correlation coefficients $r$ are negative. The discharge flow rates of Kanayama crosswalk are located below that of Otsu-dori plots. Left turning vehicles may have difficulty to find acceptable gaps quickly in Kanayama crosswalk because the crosswalk length of Kanayama is shorter than Otsu-dori, consequently pedestrians may arrive at the conflict-area within short time. Figure 7 shows the variation of discharge flow rate with increasing crosswalk length, the discharge flow rate is significantly differ by different crosswalks.

Table 2 Correlation of influencing factors with discharge flow rate

\begin{tabular}{cc}
\hline Influencing factors & Correlation \\
\hline Near-side pedestrian arrival rate rate $(\mathrm{ped} / \mathrm{sec})$ & -0.5655 \\
Far-side pedestrian arrival rate $(\mathrm{ped} / \mathrm{sec})$ & -0.3744 \\
Intersection angle & 0.1002 \\
Stop line set back distance $(\mathrm{m})$ & -0.4378 \\
Crosswalk set back distance $(\mathrm{m})$ & 0.5219 \\
Crosswalk length $(\mathrm{m})$ & 0.2833 \\
\hline
\end{tabular}

The correlation of possible influencing factors on left turning vehicles discharge flow rate is calculated to identify the significant influencing factors. Table 2 shows the result of the correlation between influencing factors and discharge flow rate. As we observe from the result near-side and far-side pedestrian arrival rates, crosswalk length, stop line setback distance and crosswalk setback distance have a significant correlation with discharge flow rate, therefore considering those factors as the influencing factor of discharge flow rate estimation is reasonable.

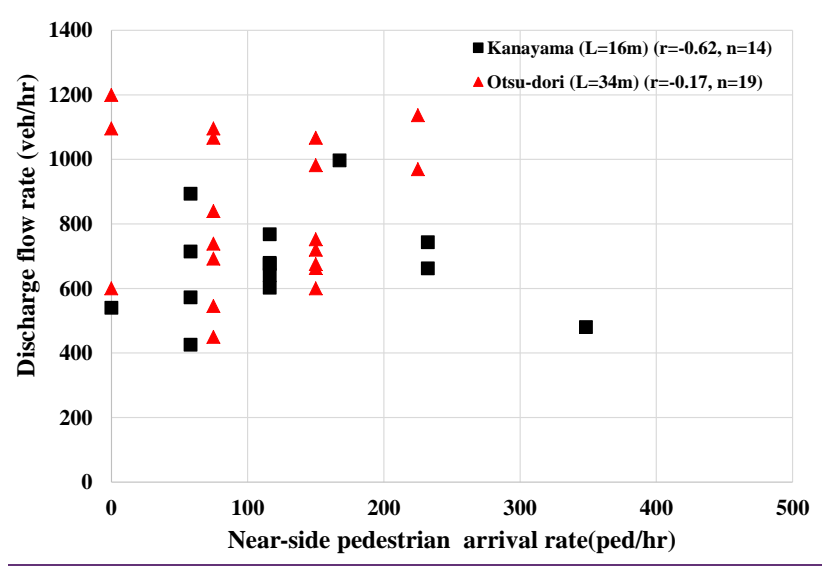

Figure 5 Observed discharge flow rate related with nearside pedestrian arrival rate

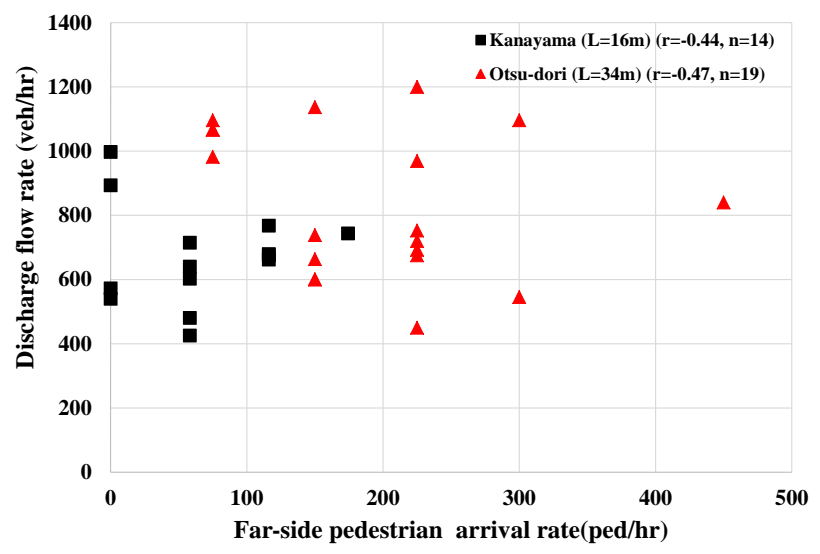

Figure 6 Observed discharge flow rate related with farside pedestrian arrival rate

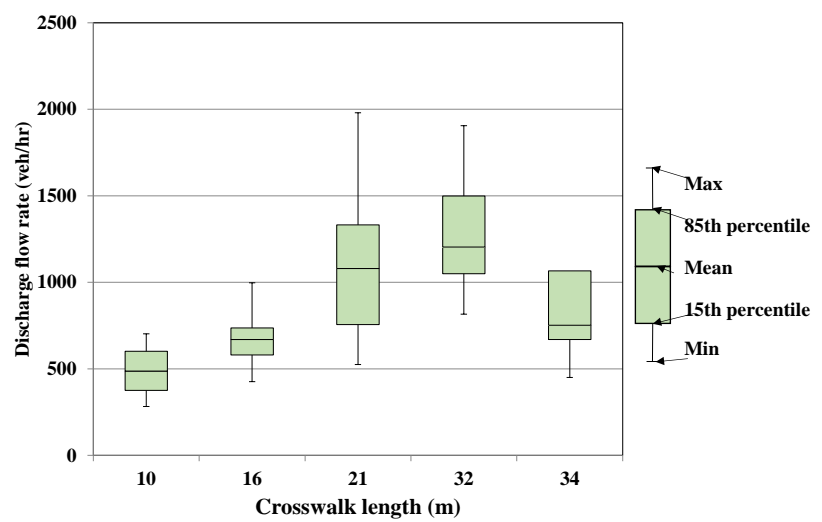

Figure 7 Variation of discharge flow rate with increasing crosswalk length

Table 3 shows the coefficients for linear regression model of discharge flow rate. For simplification reason we applied a linear regression model. Near-side and far- 
side pedestrian arrival rates have a negative impact to left turning vehicles discharge flow rate, when higher number of pedestrian's presence in the conflict-area the possibility of left turning vehicles to pass the conflict-area will be lower. Stop line set back distance has a negative impact on left turning vehicles discharge flow rate, when the stop line is far from the intersection the arriving time to the crosswalk will be long due to that there will be delay to pass the conflict-area and unused green time may increase. Consequently, with short discharge flow time the total number of left turning vehicles will be decrease.

Table 3 Coefficients of discharge flow rate model

\begin{tabular}{cccc}
\hline Variables & Coefficients & t Stat & $\mathrm{R}^{2}$ \\
\hline $\begin{array}{c}\text { Intercept } \\
\text { Near-side pedestrian } \\
\text { arrival rate (ped/hr) }\end{array}$ & -0.9449 & -3.0671 & \\
$\begin{array}{c}\text { Far-side pedestrian } \\
\text { arrival rate (ped/hr) }\end{array}$ & -1.2908 & -3.6427 & 0.53 \\
$\begin{array}{c}\text { Crosswalk set back } \\
\text { distance (m) }\end{array}$ & 16.8644 & 2.8768 & $(\mathrm{n}=97)$ \\
$\begin{array}{c}\text { Stop line set back } \\
\text { distance (m) }\end{array}$ & -18.7983 & -2.1979 & \\
$\begin{array}{c}\text { Crosswalk length (m) } \\
\text { rosth }\end{array}$ & 12.3621 & 3.0608 & \\
\hline
\end{tabular}

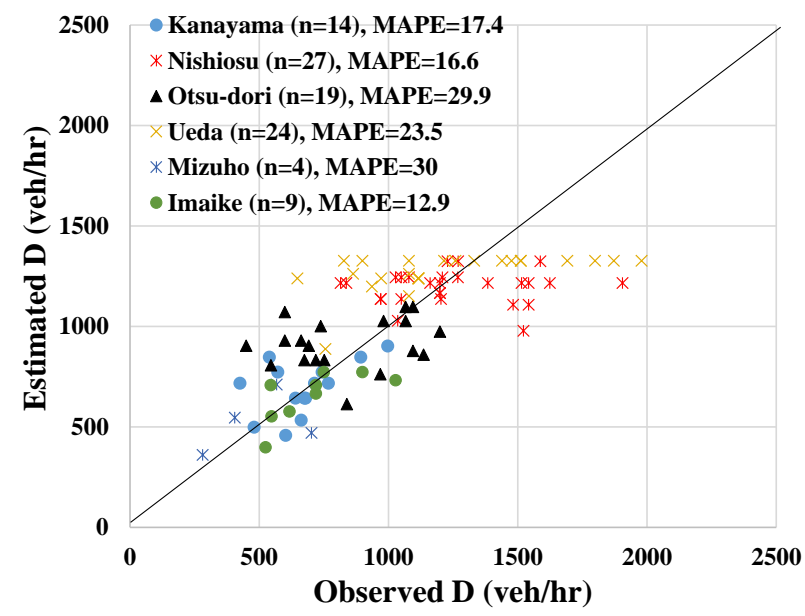

Figure 8 Left turning vehicles discharge flow rate model validation

On the other hand, crosswalk set back distance and crosswalk length have a positive impact to discharge flow rate. This implies that when the crosswalk position is near to the intersection the left turning vehicles discharge flow rate will decrease. The reason is may be related with sight distance, left turning vehicles can easily notice the crossing pedestrians at the onset of green and they will approach with lower turning speed, due to that the number of turning vehicles per cycle will decrease. In addition, for shorter crosswalks left turning vehicles may wait for longer time before they pass the conflict-area. Because, arriving pedestrians may presence on the conflict-area frequently due to short arriving time. In the model, crosswalk setback distance has higher influence on discharge flow rate compared with other variables. This implies that, even if we have lower pedestrian demand on the crosswalk, without proper crosswalk layout design the left turning vehicles discharge flow rate may decrease.
To validate the discharge flow rate model, the model estimation is compared with the observed discharge flow rates. As the validation shown in Figure 8, the proposed model can reasonably estimate the left turning vehicles discharge flow rate by considering the influence of pedestrians and different crosswalk layout. The MAPE values of each crosswalk are reasonably low, which implies that the model can estimate the observed discharge flow rate with lower error. However, the MAPE value of Mizuho crosswalk is comparatively higher, which is because of the small number of sample.

\section{ESTIMATION OF LEFT TURN LANE CAPACITY BASED ON PROPOSED METHOD}

In the above sections we tried to summarize how we can express the influence of crossing pedestrians and geometric layout, accordingly their influence on the left turn lane capacity can be generalized using Eqn. (7).

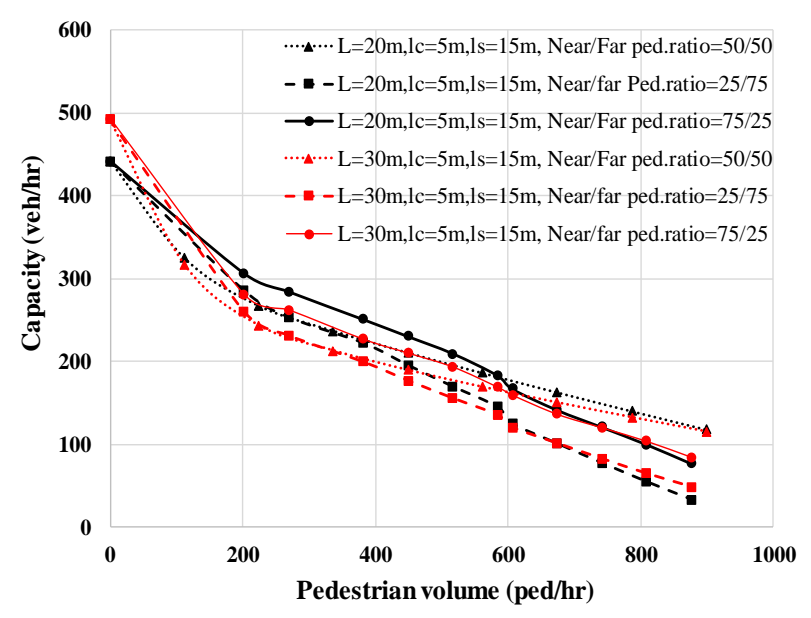

Figure 9 Estimated left turn lane capacity

Figure 9 shows a case study estimation of left turn lane capacity under two different crosswalk length and different near-side/far-side pedestrian flow proportion cases, with increasing pedestrian volume. For all conditions, when pedestrian demand increases the left turn lane capacity has a decreasing trend. The black and red color curves in Figure 9 illustrate the comparison of capacity estimation when crosswalk length varies, for low pedestrian demand condition around 0 to $200 \mathrm{ped} / \mathrm{hr}$ longer crosswalk has higher left turn lane capacity. While in the middle of the curve around $200 \mathrm{ped} / \mathrm{hr}$ to 600 $\mathrm{ped} / \mathrm{hr}$ shorter crosswalk perform around $16 \%$ higher than the other crosswalk.

Another interesting point that can be noticed from Figure 10 is the distribution of the curves after $600 \mathrm{ped} / \mathrm{hr}$, we can clearly realize that, for both crosswalks the curve with equal near-side/far-side proportion is higher than the other proportion cases. This implies that, in bi-directional pedestrian flow the opposing higher pedestrian demand will affect the crossing pedestrian's movement, thus left turning vehicles waiting time will increase. Consequently, left turn lane capacity may decrease; from the curves of Figure 10 we can notice that after $600 \mathrm{ped} / \mathrm{hr}$ there will be around $12 \%$ up to $18 \%$ increase in reduction of capacity due to bi-directional pedestrian flow interaction for $75 / 25$ and 25/75 cases compared with 50/50 case. 
Table 4 Comparison of left turn capacity estimation

\begin{tabular}{|c|c|c|c|c|c|c|c|}
\hline $\begin{array}{l}\text { Cycle } \\
\text { No. }\end{array}$ & Crosswalk & $\begin{array}{c}\text { Near-side pedestrian } \\
\text { volume } \\
(\mathrm{Ped} / \mathrm{hr}) \\
\end{array}$ & $\begin{array}{l}\text { Far-side } \\
\text { pedestrian } \\
\text { volume } \\
(\mathrm{Ped} / \mathrm{hr}) \\
\end{array}$ & $\begin{array}{l}\text { Observed } \\
\text { capacity } \\
(\mathrm{veh} / \mathrm{hr})\end{array}$ & $\begin{array}{l}\text { Proposed } \\
\text { method } \\
\text { estimation } \\
\text { (veh/hr) }\end{array}$ & $\begin{array}{c}\text { JSTE } \\
\text { estimation } \\
(\mathrm{veh} / \mathrm{hr})\end{array}$ & $\begin{array}{l}\text { HCM estimation } \\
\text { (veh/hr) }\end{array}$ \\
\hline 1 & Nishiosu & 45 & 90 & 248 & 171 & 537 & 462 \\
\hline 2 & Nishiosu & 0 & 45 & 315 & 174 & 547 & 559 \\
\hline 3 & Nishiosu & 45 & 45 & 293 & 299 & 546 & 510 \\
\hline 4 & Nishiosu & 68 & 113 & 270 & 213 & 516 & 413 \\
\hline \multirow[t]{3}{*}{5} & Nishiosu & 23 & 90 & 338 & 213 & 546 & 486 \\
\hline & & MAPE & & & 42.65 & 45.7 & 39.6 \\
\hline & & $R M S E$ & & & 94.35 & 247.2 & 197.7 \\
\hline 6 & Otsu-dori & 90 & 473 & 113 & 187 & 338 & 38 \\
\hline 7 & Otsu-dori & 90 & 225 & 158 & 180 & 408 & 289 \\
\hline 8 & Otsu-dori & 68 & 315 & 180 & 194 & 386 & 220 \\
\hline \multirow[t]{3}{*}{9} & Otsu-dori & 113 & 338 & 113 & 136 & 370 & 152 \\
\hline & & $M A P E$ & & & 19.24 & 62.7 & 71,5 \\
\hline & & $R M S E$ & & & 41.1 & 235.6 & 80.5 \\
\hline 10 & Ueda & 0 & 135 & 315 & 209 & 595 & 574 \\
\hline 11 & Ueda & 0 & 0 & 383 & 432 & 698 & 698 \\
\hline 12 & Ueda & 0 & 23 & 405 & 417 & 629 & 677 \\
\hline \multirow[t]{3}{*}{13} & Ueda & 68 & 68 & 383 & 359 & 595 & 574 \\
\hline & & MAPE & & & 17.8 & 40.8 & 40.9 \\
\hline & & $R M S E$ & & & 59.6 & 261 & 263.1 \\
\hline 14 & Imaike & 135 & 180 & 158 & 141 & 488 & 444 \\
\hline 15 & Imaike & 90 & 135 & 203 & 203 & 535 & 441 \\
\hline \multirow[t]{5}{*}{16} & Imaike & 180 & 113 & 158 & 114 & 500 & 371 \\
\hline & & MAPE & & & 16.5 & 66.1 & 58.7 \\
\hline & & $R M S E$ & & & 26.6 & 334.8 & 248.3 \\
\hline & & Total MAPE & & & 24.3 & 56.6 & 51 \\
\hline & & Total RMSE & & & 61.6 & 287.5 & 221.4 \\
\hline
\end{tabular}

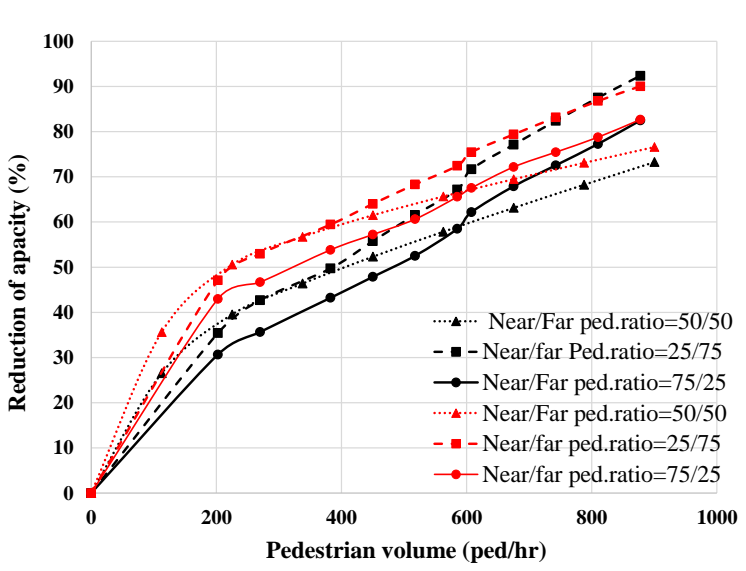

Figure 10 Percentage of capacity reduction

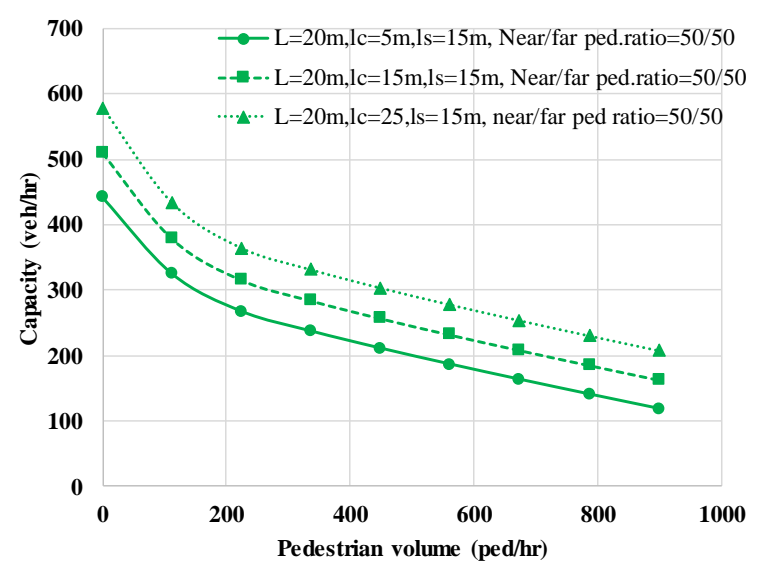

Figure 11 Sensitivity of left turn lane capacity for crosswalk set back distance
The sensitivity of the left turn lane capacity for change in crosswalk set back distance is shown in Figure 11. The increase in crosswalk set back distance will raise the left turn lane capacity approximately by $17 \%$ under all pedestrian demand conditions.

\section{COMPARISON OF PROPOSED METHOD CAPACITY ESTIMATION WITH EXISTING MANUALS ESTIMATIONS}

The proposed capacity estimation method results are compared with two existing capacity estimation procedures, i.e. Guide for Planning, Design and Traffic Signal Control of Japan (JSTE manual) and Highway capacity manual (HCM 2016). Table 4 shows the summary of the comparison between the estimation methods. In addition, the estimation methods compared with observed capacity, the observed capacity calculated based on observed over saturated cycles of different crosswalks. The HCM method proposes adjustment factors based on the pedestrian volume and then the capacity is estimated by adjusting the base saturation flow rate. While the JSTE method similarly propose adjustment factors to adjust the base saturation flow rate, However, the JSTE method consider crosswalk length as an additional factor to propose adjustment factors. Based on the comparison results, JSTE and HCM methods overestimate left turn lane capacity, while proposed method capacity estimation is reasonable compared with the others. The reason behind this variation in capacity estimation is related with;

- Proper bi-directional pedestrian flow consideration especially in the higher pedestrian demand conditions, 
- Consideration of the influence of crosswalk layout on the left turning vehicles and crossing pedestrians movement and,

- The other important factor may be associated with the proposed base saturation flow rate of $1800 \mathrm{pcu} / \mathrm{hr}$ for left turning vehicles by JSTE and HCM seemed higher to use for left turn lane capacity estimation.

Additionally, in order to quantitatively evaluate the relative margin of estimation errors, Mean Absolute Percentage Error (MAPE) and Root Mean Squared Error (RMSE) are computed for all methods. For all crosswalks the RMSE and MAPE values of the proposed method is lower than that of JSTE and HCM method, this revealed that the proposed method contemplates additional relevant influencing factors to realistically estimate left turn lane capacity under different pedestrian demand and crosswalk layout conditions.

\section{CONCLUSIONS}

In this study, the left turn lane capacity estimation procedure is proposed by considering the influence of crossing pedestrians and geometric layout of the crosswalk. As the behavior of waiting pedestrians and arriving pedestrians are different, in this study we considered a separated observation for waiting pedestrians and arriving pedestrians. Waiting pedestrians have a group movement characteristic which leads to platoon dispersion behavior and bi-directional pedestrian flow interaction. While arriving pedestrians, most of the time, passes the crosswalk individually. The waiting pedestrian's behavior is captured by pedestrian presence probability model which considers crosswalk layout condition and bi-directional pedestrian flow situations, and then the pedestrian presence-time is estimated based on the model. Additionally, the left turning vehicles discharge flow rate is observed and modeled by considering the influence of crosswalk length, crosswalk setback distance, stop-line set back distance and pedestrian arrival rate. Finally, all the above models combined to estimate the left turn lane capacity.

In general, the proposed left turn lane capacity estimation method is reasonable and significant to evaluate the performance of signalized crosswalks or as overall to evaluate the performance of the signalized intersections. The comparison with existing methods revealed that the proposed method can improve the capacity estimation procedure by taking into account the realistic left turning vehicles and pedestrian's movement conditions.

\section{REFERENCES}

[1] Transportation Research Board, Highway Capacity Manual (HCM), National Research Council, Washington, D.C, U.S.A, 2016.

[2] Japan Society of Traffic Engineers (JSTE), "Planning and Design of at-grade Intersections Basic Edition -; Guide for Planning, Design and Traffic Signal Control of Japan”, 2018. (in Japanese)

[3] Lu X., Wang J., "Analysis of the Right-turn lane Capacity under the influence of pedestririans", $19^{\text {th }}$
COTA International Conference of Transportation, 2019.

[4] Zhang, W., Yang, X. and Xu, Z., "Study on the Capacity of Right-Turn Movement under Pedestrians' Influence at Signalized Intersections", Proceedings of international Conference of Chinese Transportation Professionals (ICCTP, 2012).

[5] Chen, Y., He, Y. and Sun, X., "Impact of pedestrian traffic on saturation rate of protected left-turn at urban intersections", Open Journal of Applied Sciences, pp. 22-31, 2015.

[6] Roshani M. and Bargegol I "Effect of Pedestrians on the Saturation Flow Rate of Right Turn Movements at Signalized Intersection - Case Study from Rasht City", WMCAUS IOP Publishing IOP Conference Series: Materials Science and Engineering, 245, 2017.

[7] Nassereddine H., Kelvin R. and Noyce D., "Modeling Vehicle-Pedestrian Interactions Using a Non-Probabilistic Regression Approach", Transportation Research Record, vol 2675, Issue 1, 2021.

[8] Iasmin H, Kojima A., and Kubota H., "Yielding behavior of left turning driver towards pedestrian/cyclist: Impact of intersection angle", Journal of the Eastern Asia Society for Transportation Studies, vol.11, 2015.

[9] Akash, J., Ankit, G., Rajat, R., "Pedestrian Crossing Behavior Analysis at Intersections", International Journal of Traffic and Transportation Engineering, vol. 4, no. 1, pp.103-116, 2014.

[10] Muleya, D., Alhajyaseena, W., Kharbechea, M., AlSalem, M., "Pedestrians' Speed Analysis at Signalized Crosswalks", The 9th International Conference on Ambient Systems, Networks and Technologies, Procedia Computer Science 130 (2018) pp. 567-574, 2018.

[11] Park, J, Yang W, Yu W, Wanger I., Ahemed S., “An investigation of pedestrian crossing speed at signalized intersections with heavy pedestrian volumes", Transportation Research Board Annual Meeting, 2014.

[12] Zhang X. and Nakamura H., "Analytical study on the relationship between Level of Pedestrian-vehicle separation and performance of signalized intersections", Journal of Japan Society of Traffic Engineers, vol.3, no.5, pp. 11-20, 2017. (in Japanese).

[13] Emagnu Y., Zhang X., Iryo-Asano $M$ and Nakamura $\mathrm{H}$, "Estimation of expected pedestrian presence-time at the conflict-area of signalized crosswalks", Journal of Japan Society of Traffic Engineers, Ser.D3, vol.76, no. 5, 2020.

[14] Suzuki, K. and Nakamura, H., "Traffic Analyzer The Integrated Video Image Processing System for Traffic Flow Analysis", Proceedings of the 13th World Congress on Intelligent Transportation Systems, 2006. 